CLINICAL PRACTICE

Clinical Vignettes

\title{
An Elusive Case of Mycosis Fungoides: Case Report and Review of the Literature
}

\author{
Vincent A. Pallazola, $M D^{7}$, Gerard Deib, MD' , Soni Abha, DO, MPH , \\ Rabih M. Geha, $M D^{4,5}$, and Kimiyoshi Kobayashi, MD, $M B A^{7}$
}

\begin{abstract}
'Department of Medicine, Johns Hopkins University School of Medicine, Baltimore, MD, USA; '2Department of Radiology, Johns Hopkins University School of Medicine, Baltimore, MD, USA; ${ }^{3}$ Department of Dermatology, Johns Hopkins University School of Medicine, Baltimore, MD, USA;

${ }^{4}$ Department of Medicine, University of California San Francisco, San Francisco, CA, USA; ${ }^{5}$ Medical Service, San Francisco Veterans Affairs Medical Center, San Francisco, CA, USA.
\end{abstract}

Erythroderma refers to a spectrum of skin diseases resulting in diffuse erythema and scaling encompassing $\geq 90 \%$ of the body surface area. The differential diagnosis ranges from primary dermatologic diseases such as atopic dermatitis and psoriasis to potentially deadly causes such as staphylococcal toxic shock syndrome, toxic epidermal necrolysis, and malignancy. Cutaneous T cell lymphoma (CTCL) is an uncommon but highly morbid cause of erythroderma. This non-Hodgkin lymphoma remains a diagnostic challenge due to its variable clinical presentation and varied histologic features. Mycosis fungoides (MF) is the most common form of CTCL. Making a timely diagnosis is challenging as it may mimic inflammatory diseases of the skin including eczema, psoriasis, lichen planus, and cutaneous lupus. We present a case of a 58year-old man who presented with 5 years of cutaneous symptoms and several months of fevers and night sweats, ultimately diagnosed as MF. Owing to diffuse CD30 positivity, he was a candidate for brentuximab vedotin, an antibody-drug conjugate medication that selectively targets the CD30 antigen. This resulted in an excellent therapeutic response.

KEY WORDS: cancer; dermatology; clinical image.

$\mathrm{J}$ Gen Intern Med 34(11):2669-74

DOI: $10.1007 / \mathrm{s} 11606-019-05231-\mathrm{z}$

(c) Society of General Internal Medicine 2019

\section{CASE PRESENTATION}

A 58-year-old man presented to the emergency department with a progressive pruritic and painful erythematous rash covering his entire body. Five years earlier, he developed an intermittent burning sensation in his skin that lasted several months and progressed to multiple ulcerations on the dorsal and plantar aspects of his right foot. He later noted a scaly, pruritic, and painful rash on his torso. His lesions fluctuated between multiple stages of discoloration, ulceration, scaling, and scarring. Pruritus and pain were exacerbated by cold

There were no prior presentations or publications of the content below.

Received October 19, 2018

Revised April 30, 2019

Accepted July 9, 2019

Published online August 6, 2019 exposure and alleviated by warmth and direct sunlight. Several months before admission, he developed drenching night sweats and chills. Since the onset 5 years ago, he underwent numerous evaluations that included a rapid plasma reagin (RPR) that was repeatedly negative and skin biopsies with inconclusive results. He was treated with several topical steroids, antifungal creams, and oral antibiotics in addition to general wound care without significant improvement.

His medical history included chronic hepatitis B infection without cirrhosis, untreated latent tuberculosis, and treated latent syphilis in the 1990s. He took no medications or supplements. He was born in Haiti and moved to Maryland 4 years prior to presentation. He did not smoke tobacco, use alcohol or illicit substances, and had one sexual partner for the last 5 years. He previously worked in a meat packing plant, but had quit due to his inability to tolerate the cold temperatures of the freezer.

Vital signs were normal, and physical exam showed a heterogeneous, erythematous rash that spanned nearly $100 \%$ of his body surface area (Fig. 1). There was no organomegaly. He had moderately enlarged, bilateral non-tender mobile cervical, submandibular, axillary, and inguinal lymphadenopathy.

Laboratory studies were notable for an erythrocyte sedimentation rate of $68 \mathrm{~mm} / \mathrm{h}$ (normal, $1-20 \mathrm{~mm} / \mathrm{h}$ ) and a Creactive peptide of $4.6 \mathrm{mg} / \mathrm{dL}$ (normal, $<0.5 \mathrm{mg} / \mathrm{dL}$ ). The total white blood cell count was normal; eosinophil count was $540 \mathrm{~K} / \mathrm{mm}^{3}$ (normal, $120-300 \mathrm{~K} / \mathrm{mm}^{3}$ ). A comprehensive metabolic panel was normal, without evidence of liver function abnormalities. Human immunodeficiency virus antibodies were negative. Fluorescent treponemal antibodies were positive, RPR was negative, and treponemal pallidum particle agglutination assay was positive. Hepatitis B core and surface antibodies were positive, and surface antigen was negative. Computed tomography demonstrated multiple, symmetrically enlarged lymph nodes in the cervical, axillary, subpectoral, iliac, and inguinal regions.

Skin biopsies (Fig. 2) showed large atypical lymphocytes in the mid-dermis with $>25 \%$ large cell transformation suggestive of cutaneous T cell lymphoma (CTCL). Immunohistochemical staining was positive for $\mathrm{CD} 3, \mathrm{CD} 4$, and CD30, and negative for $\mathrm{CD}$ 7. Polymerase chain reaction (PCR) of the 

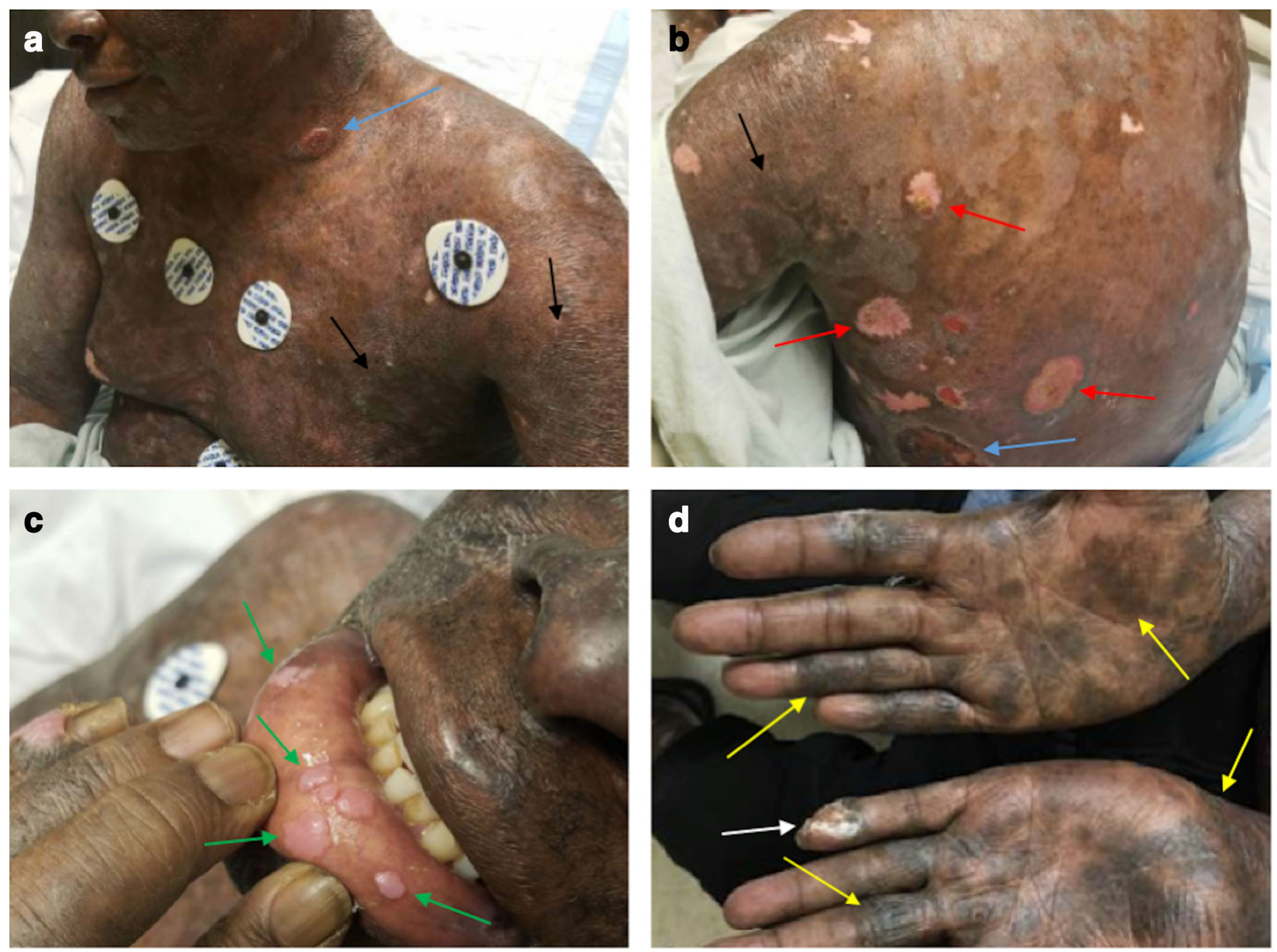

Figure 1 Panels a and b demonstrate scattered hyperpigmented patches and plaques (black arrows), punched-out ulcerations (red arrows), and subcutaneous nodules (blue arrows) in various stages of evolution. Panel c demonstrates five well-defined pink papules with irregular borders on the inner lower lip (green arrows). Panel d demonstrates thick hyperpigmented, hyperkeratotic plaques and patches on the palmar surface of the hands (yellow arrows) in addition to a superficial ulceration at the palmar aspect of the left fifth digit (white arrows). All photographs were taken upon initial presentation.

skin was positive for a $\mathrm{T}$ cell receptor gamma gene rearrangement. Peripheral flow did not detect Sézary cells, and human T-lymphotropic virus (HTLV) type I PCR was negative. Positive emission tomography scan demonstrated fluorodeoxyglucose uptake in the axillary and pelvic lymph nodes concerning for active nodal involvement; however, lymph node biopsy was deferred by the patient (Fig. 3). These findings were diagnostic of at least stage IIIa mycosis fungoides (MF) given his clinical erythroderma in the absence of visceral or blood involvement (T4NxV0B0); presence of advanced lymph node involvement (N3) could not be ruled out without lymph node biopsy in this case, which would have implicated stage $\mathrm{IVA}_{2}$ disease.

\section{DISCUSSION}

Erythroderma, commonly known as exfoliative dermatitis, is defined as diffuse erythema and scaling involving $\geq 90 \%$ of the body surface area, occurring with an annual incidence of 1 per 100,000 in the adult population. ${ }^{1}$ Exacerbation of preexisting inflammatory dermatosis such as psoriasis or atopic dermatitis accounts for the majority of cases. Hypersensitivity drug reactions are the second most common cause. Contact dermatitis, lichen planus, systemic lupus erythematosus, and staphylococcal scalded skin syndrome have also been implicated. In a patient traveling to Haiti, tropical diseases such as dengue fever and vibrio vulnificus infection are additional considerations. Although rare, case reports have associated erythroderma with fulminant viral hepatitis. ${ }^{2}$

The approach to erythroderma relies on identifying a history of inflammatory skin disease and careful evaluation of current and recent medications, especially major culprit drugs such as penicillin, sulfonamides, allopurinol, and antiepileptic drugs (e.g., phenytoin). ${ }^{3}$ Secondary syphilis was considered in this patient given that syphilis is endemic in Haiti and we could not confirm his prior treatment with their health department. Furthermore, false negative RPR can be seen in the setting of the 

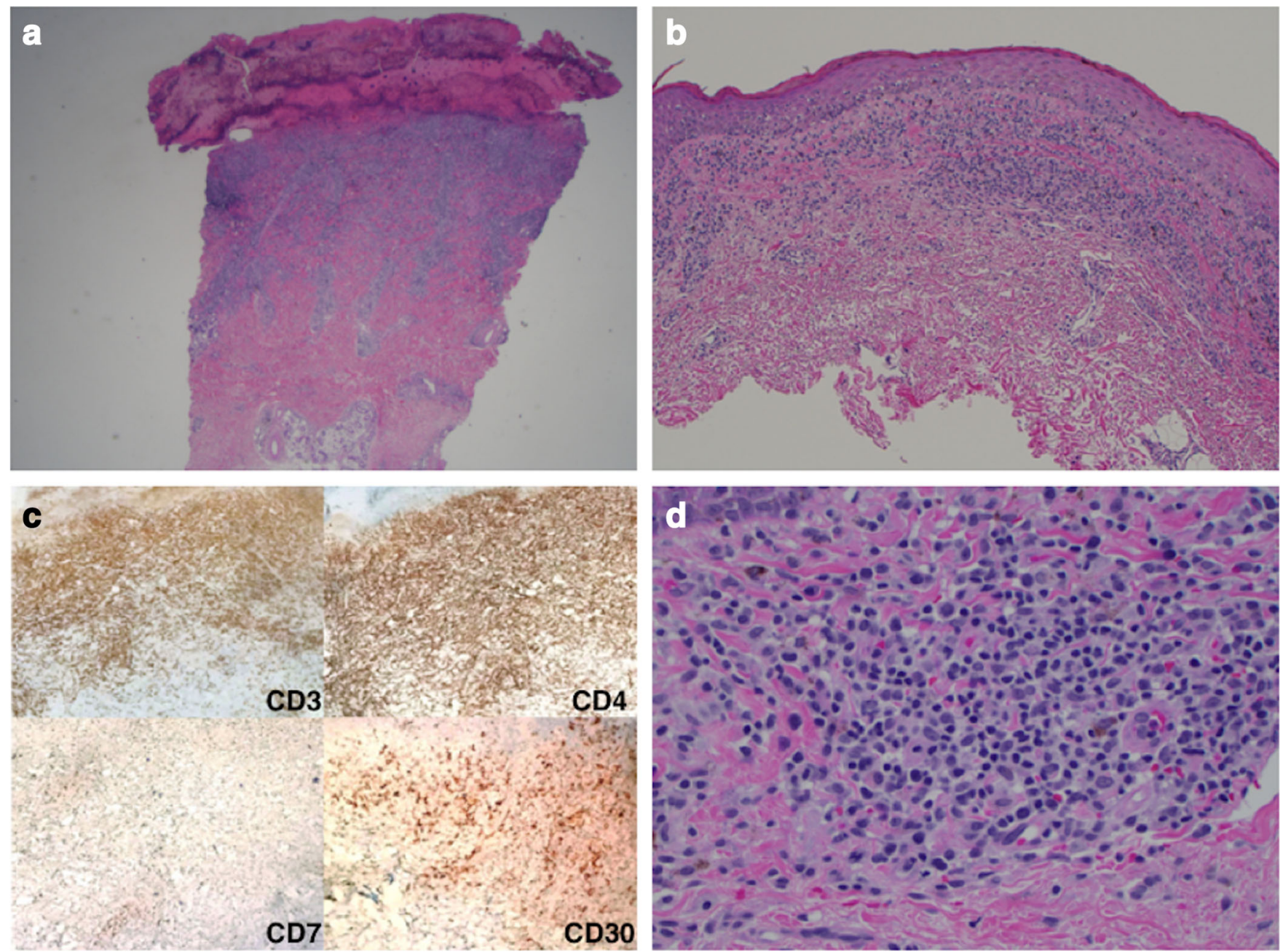

Figure 2 Hematoxylin- and eosin-stained sections of a right arm skin lesion demonstrated at $\times 20$ (a) and $\times 100$ (b) show an ulcerated epidermis with a dense infiltrate of predominantly large atypical lymphocytes within the superficial and mid portions of the dermis. The atypical lymphoid infiltrate is immunoreactive for $\mathrm{CD3}, \mathrm{CD} 4$, with a loss of $\mathrm{CD} 7$, and prominent $\mathrm{CD30}$ positivity $(\mathrm{c} ; \times 200)$. The increased number of large atypical lymphoid cells $(\mathrm{d} ; \times \mathbf{4 0 0})$ exceeded the diagnostic threshold for large cell transformation $(>25 \%)$.

prozone reaction, where exceptionally high titers cause interference with the formation of antigen-antibody lattice. Malignant syphilis, or lues maligna, is a severe form of secondary syphilis that presents as an ulcerative predominant erythroderma. ${ }^{4}$ Malignant syphilis was commonly observed in the seventeenth century prior to the development of antibiotics, ${ }^{5}$ and has recently re-emerged with the HIV epidemic.

In up to $30 \%$ of cases of erythroderma, a clear etiology is not identified. Certain exam findings may be useful in differentiating etiology of erythroderma. Nail changes (e.g., onycholysis) suggest psoriatic erythroderma. ${ }^{6}$ Palmoplantar keratoderma seen in this patient is often observed in pityriasis rubra pilaris and CTCL. ${ }^{7}$ Eosinophilia suggests a drug reaction (e.g., drug-induced hypersensitivity syndrome), hypereosinophilic syndrome, or peripheral $\mathrm{T}$ cell lymphoma. In the absence of a history of inflammatory dermatitis or a clear culprit drug, a skin biopsy is often required.

CTCL is a rare cause of erythroderma, with a prevalence of less than $5 \%$ among all cases of erythroderma. ${ }^{8}$ Incidence of CTCL has increased over time, with one recent systematic review estimating annual incidence of 0.39-0.9 per one million. ${ }^{9} \mathrm{MF}$ is an extranodal, indolent non-Hodgkin lymphoma and is the most common cause of CTCL. In contrast, Sézary syndrome represents a more aggressive, leukemic variant of CTCL marked by a high number of circulating malignant cells (Sézary cells) in the peripheral blood and clonal TCR rearrangement accompanied by erythroderma. It is thought that infection via HTLV-I/II, which is endemic to the Caribbean, Japan, South American, and Africa, provokes malignant transformation of $\mathrm{T}$ cells in the majority of cases of $\mathrm{CTCL}^{10}$; although affected patients are commonly antibody negative, presence of HTLV DNA fragments via Western blot was detected in $92 \%$ of patients in one study. ${ }^{11}$ This case highlights the elusive nature of MF, as the patient was evaluated by multiple physicians with a series of indeterminate skin biopsies.

The presentation of isolated skin MF can be extremely difficult due to its ability to mimic a wide range of diseases. CTCL may present with a variety of clinical 

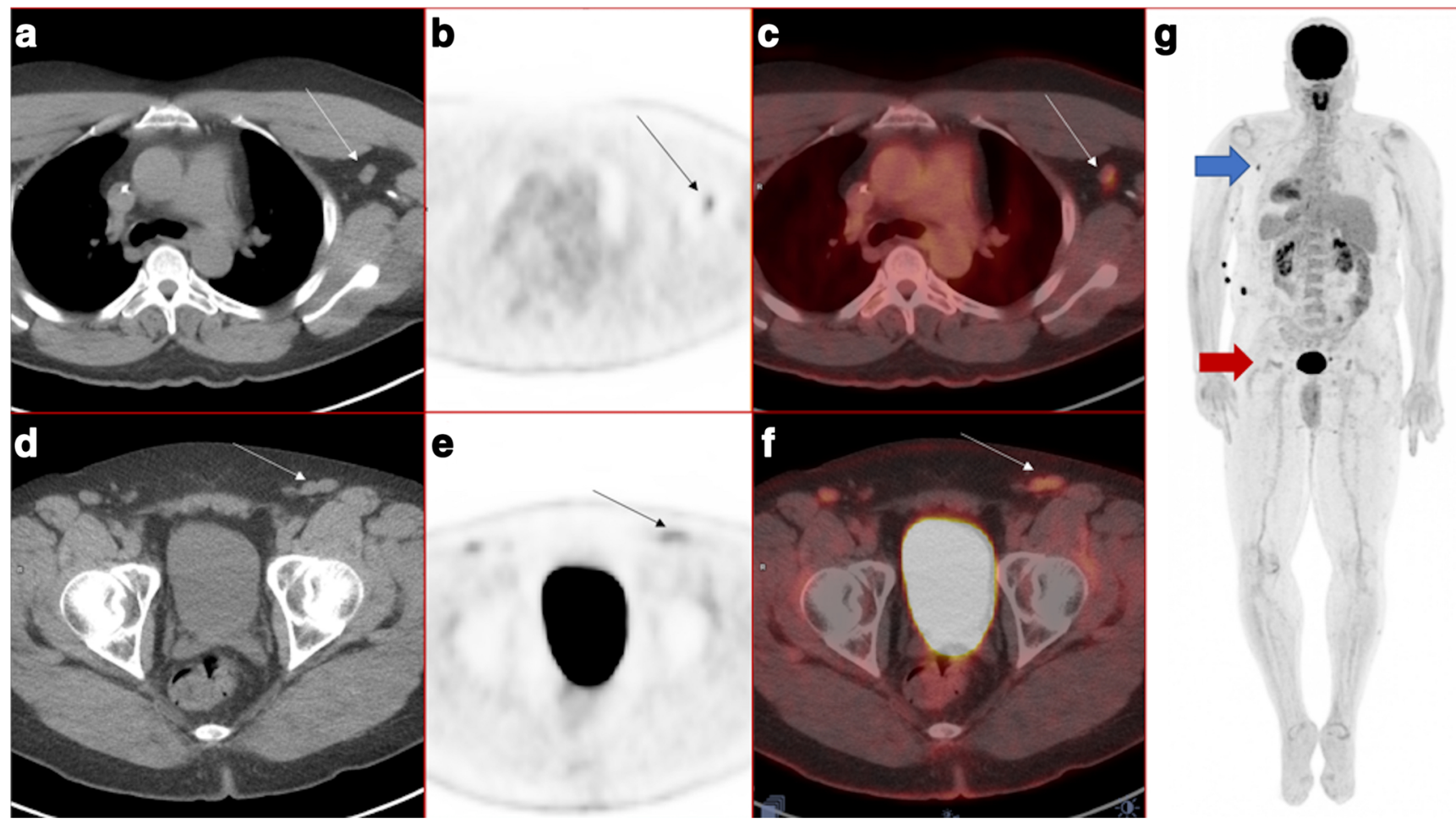

Figure 3 Axial computerized tomography (CT) (panel a), standardized uptake value positron emission tomography (SUV PET) (panel b), and fused PET-CT (panel c) images through the upper thorax demonstrate a hypermetabolic left axillary lymph node (black and white arrows). Axial CT (panel d), SUV PET (panel e), and fused PET-CT (panel f) images through the pelvis demonstrate hypermetabolic left inguinal lymph nodes (black and white arrows). Maximum intensity projection (MIP) whole body image (panel g) demonstrates hypermetabolic lymph nodes, including the left axillary (blue arrow) and the left inguinal (red arrow) to good effect.

and histopathological variants, contributing to misdiagnosis and delay in identification. Diagnosis may be further confounded by partial treatment of malignant cells with empiric topical immunosuppressive therapies. Patients should have a close follow-up to detect progression of lesions, lymphadenopathy, and constitutional symptoms. Repeated biopsies from multiple sites are often needed to increase diagnostic yield. ${ }^{8}$ One study showed a median time of 4 years from the onset of the first skin lesion to diagnosis in patients with cutaneous-limited disease. ${ }^{12}$ The International Society for Clinical Lymphoma proposed an algorithm for diagnosis of early MF that utilizes a variety of clinical, histopathologic, and immunopathologic criteria that can be applied after obtaining a skin biopsy. ${ }^{13}$ If a diagnosis of CTCL is entertained, physicians should obtain consultation with a dermatopathologist with experience in diagnosing cutaneous lymphoma. ${ }^{8}$

The management of CTCL depends on the staging of disease, combining various symptomatic treatments (e.g., emollients, antipruritics) with specific topical and systemic treatments. ${ }^{14} \mathrm{CTCL}$ predisposes patients to viral and bacterial infections due to skin ulceration and impaired cellular and humoral immunity. ${ }^{15}$ In particular, disseminated herpes simplex virus and varicella zoster virus have been described in patients with advanced disease with extracutaneous involvement and in those undergoing chemotherapy. ${ }^{16,}{ }^{17}$ Appropriate wound care should be proactively implemented, and routine skin exams should be performed to evaluate for infectious complications.

Stage I-IIA disease is typically managed with ultraviolet B therapy, local electron beam therapy, and topical therapies such as corticosteroids, nitrogen mustard, and imiquimod. Patients with stage IIB-IV disease without leukemic involvement receive systemic methotrexate or bexarotene in addition to the above treatments, whereas those with blood involvement receive single agent or combination chemotherapy with methotrexate, pegylated doxorubicin, gemcitabine, and purine analogs. Prognosis for CTCL is variable, ranging from near-normal survival in stage Ia patients to 13 months in patients with stage IV disease. ${ }^{18}$ Mortality is higher in patients with CD30 negativity, folliculotropic MF (a rare variant involving hair follicles), generalized skin lesions (multiple lesions in $>1$ anatomic region), and/or extracutaneous transformation. ${ }^{19}$

Given our patient's CD30 positivity, he was a candidate for brentuximab vedotin, an antibody-drug conjugate medication that selectively targets the CD30 antigen and has been shown to improve outcomes compared with conventional methotrexate or bexarotene therapy. ${ }^{20} \mathrm{He}$ was ultimately initiated on brentuximab while inpatient. Latent tuberculosis was concurrently treated with isoniazid. Although the patient reported a history of syphilis treatment in Haiti and prozone reaction was ruled out by our 

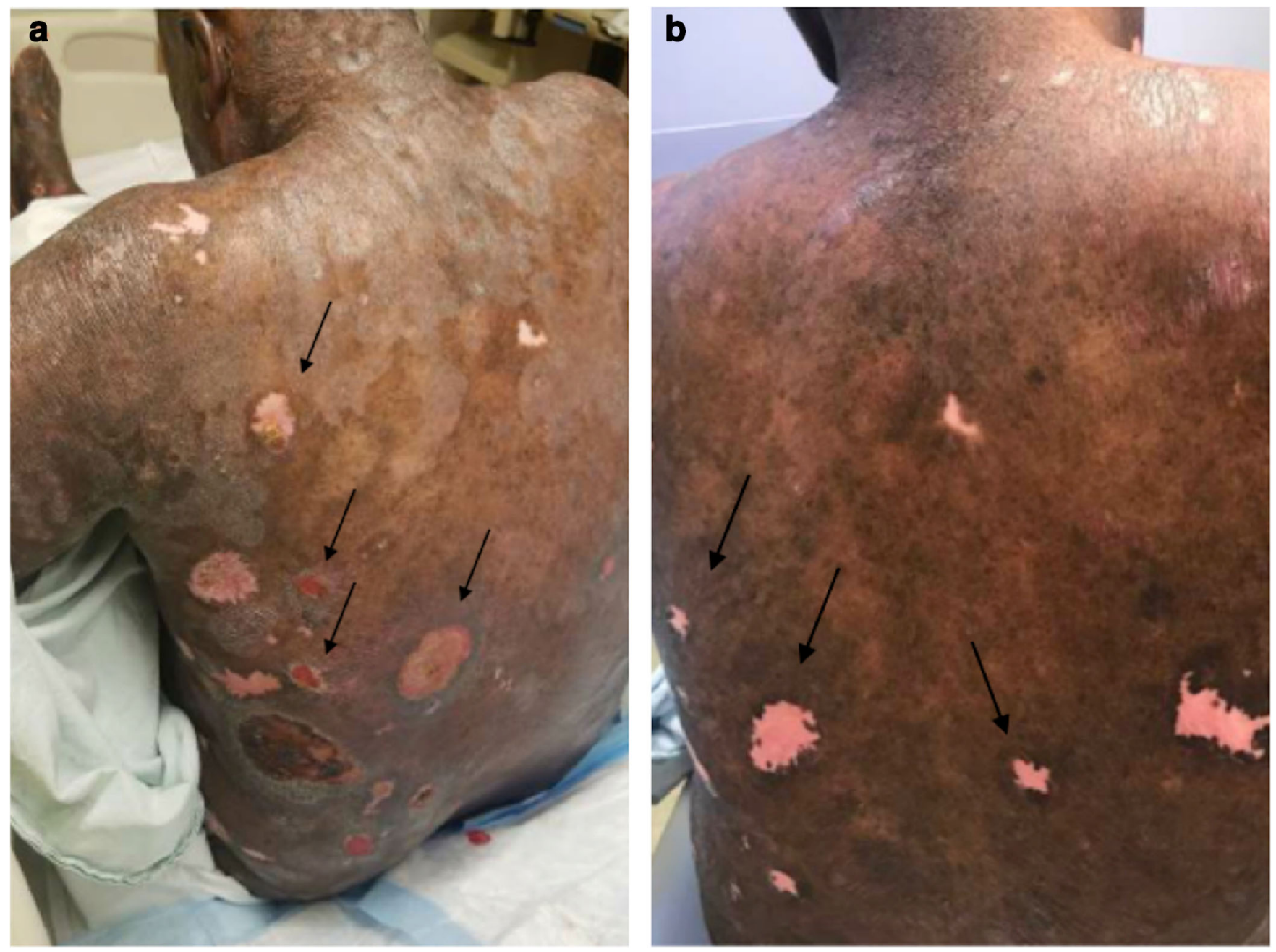

Figure 4 After three cycles of immunotherapy, the patient was noted to demonstrate significant improvement in terms of his diffuse skin lesions, as demonstrated by interval improvement in punched-out ulcerations (black arrows). Panel a represents a photograph taken upon initial presentation, and panel $b$ represents interval photography at 4-month follow-up.

laboratory, he was empirically treated with intramuscular benzathine penicillin for 3 weeks for possible late latent syphilis. After three cycles, he was noted to have significant recession of punched-out ulcerations (Fig. 4) and hyperpigmented patches and plaques as well as improvement in pain and pruritis.

\section{CONCLUSION}

Mycosis fungoides, although rare, requires a high degree of clinical suspicion. Cutaneous-limited disease is associated with excellent outcomes if promptly identified and treatment is initiated. Immunophenotyping is important in management of CTCL, as novel immunotherapies such as the CD30 antibody-drug conjugate may allow for more effective targeted therapy without the toxicities associated with conventional chemotherapy.
Corresponding Author: Vincent A. Pallazola, MD; Department of MedicineJohns Hopkins University School of Medicine, $600 \mathrm{~N}$. Wolfe Street, Baltimore, MD 21287, USA (e-mail: vpallaz1@jhmi.edu).

\section{Compliance with Ethical Standards:}

Conflict of Interest: The authors declare that they do not have a conflict of interest.

\section{REFERENCES}

1. Sigurdsson V, Steegmans PH, van Vloten WA. The incidence of erythroderma: a survey among all dermatologists in The Netherlands. $J$ Am Acad Dermatol. 2001;45(5):675-678. doi:https://doi.org/10.1067/ mjd.2001.116224

2. Schwartz RA, Leevy CM, Cohen PJ, Lambert WC. Erythroderma with fulminant hepatitis: a possible association. Cutis. 1986;37(1):56-58.

3. Rym BM, Mourad M, Bechir Z, et al. Erythroderma in adults: a report of 80 cases. Int $J$ Dermatol. 2005;44(9):731-735. doi:https://doi.org/10. $1111 /$ j.1365-4632.2004.02100.x 
4. Yamashita M, Fujii Y, Ozaki K, et al. Human immunodeficiency viruspositive secondary syphilis mimicking cutaneous T-cell lymphoma. Diagn Pathol. 2015;10:185. doi:https://doi.org/10.1186/s13000-015-0419-5

5. Ortigosa YM, Bendazzoli PS, Barbosa AM, Ortigosa LCM. Early malignant syphilis. An Bras Dermatol. 2016;91(5 Suppl 1):148-150. doi:https://doi. org/10.1590/abd1806-4841.20164491

6. Haneke E. Nail psoriasis: clinical features, pathogenesis, differential diagnoses, and management. Psoriasis (Auckl). 2017;7:51-63. doi:https://doi.org/10.2147/PTT.S126281

7. Patel S, Zirwas M, English JC. Acquired palmoplantar keratoderma. Am J Clin Dermatol. 2007;8(1):1-11.

8. Vonderheid EC. On the diagnosis of erythrodermic cutaneous T-cell lymphoma. J Cutan Pathol. 2006;33 Suppl 1:27-42. doi:https://doi.org/ 10.1111/j.0303-6987.2006.00541.x

9. Dalal MR, Mitchell SA, McCloskey C, Zagadailov EA, Gautam A Epidemiological and humanistic burden of cutaneous T-cell lymphomas: results of a systematic review. Hematological Oncology. 2017;35(S2):389390. doi:https://doi.org/10.1002/hon.2439_154

10. Sakamoto FH, Colleoni GWB, Teixeira SP, et al. Cutaneous T-cell lymphoma with HTLV-I infection: clinical overlap with adult T-cell leukemia/lymphoma. International Journal of Dermatology. 2006;45(4):447-449. doi:https://doi.org/10.1111/j.1365-4632.2006. 02687.x

11. Pancake BA, Zucker-Franklin D, Coutavas EE. The cutaneous T cell lymphoma, mycosis fungoides, is a human $\mathrm{T}$ cell lymphotropic virusassociated disease. A study of 50 patients. J Clin Invest. 1995;95(2):547554

12. van Doorn R, Van Haselen CW, van Voorst Vader PC, et al. Mycosis fungoides: disease evolution and prognosis of 309 Dutch patients. Arch Dermatol. 2000;136(4):504-510.

13. Pimpinelli N, Olsen EA, Santucci M, et al. Defining early mycosis fungoides. J Am Acad Dermatol. 2005;53(6):1053-1063. doi:https://doi. org/10.1016/j.jaad.2005.08.057
14. Willemze R, Hodak E, Zinzani PL, Specht L, Ladetto M, ESMO Guidelines Working Group. Primary cutaneous lymphomas: ESMO Clinical Practice Guidelines for diagnosis, treatment and follow-up. Ann Oncol. 2013;24 Suppl 6:vi149-154. doi:https://doi.org/10.1093/annonc/mdt242

15. Krejsgaard T, Odum N, Geisler C, Wasik MA, Woetmann A. Regulatory T cells and immunodeficiency in mycosis fungoides and Sézary syndrome. Leukemia. 2012;26(3):424-432. doi:https://doi.org/10.1038/leu.2011. 237

16. Vonderheid EC, van Voorst Vader PC. Herpes zoster-varicella in cutaneous T-cell lymphomas. Arch Dermatol. 1980;116(4):408-412.

17. Axelrod PI, Lorber B, Vonderheid EC. Infections complicating mycosis fungoides and Sézary syndrome. JAMA. 1992;267(10):1354-1358.

18. Arulogun SO, Prince HM, Ng J, et al. Long-term outcomes of patients with advanced-stage cutaneous T-cell lymphoma and large cell transformation. Blood. 2008;112(8):3082-3087. doi:https://doi.org/10.1182/blood2008-05-154609

19. Benner MF, Jansen PM, Vermeer MH, Willemze R. Prognostic factors in transformed mycosis fungoides: a retrospective analysis of 100 cases. Blood. 2012;119(7):1643-1649. doi:https://doi.org/10.1182/blood2011-08-376319

20. Prince HM, Kim YH, Horwitz SM, et al. Brentuximab vedotin or physician's choice in CD30-positive cutaneous T-cell lymphoma (ALCANZA): an international, open-label, randomised, phase 3, multicentre trial. Lancet. 2017;390(10094):555-566. doi:https://doi. org/10.1016/S0140-6736(17)31266-7

Publisher's Note Springer Nature remains neutral with regard tojurisdictional claims in published maps and institutional affiliations. 